\section{Is a POMMP approach the way to move the medication safety agenda forward in your organisation}

\author{
Steven D Williams
}

Is a POMMP approach the way to move the medication safety agenda forward in your organisation?

So, you are back at work after attending the 20th European Association of Hospital Pharmacy (EAHP) conference in Hamburg. What did you learn about patient safety, and more importantly, what are you doing now or trying to do differently at your workplace to improve medication safety?

One suggestion is to take a POMMP approach to improving medication safety in your organisation.

The first $P$ is obvious, it is about Patients. The opening plenary by Peggy Maguire reminded us about the need for real patient engagement and the dangers of not involving patients in their own healthcare decisions. For example, a box of co-amoxiclav tablets, simply preprinted with the words 'contains penicillin', given to a patient to self-medicate, may stop a patient with a known penicillin allergy from popping the pill in his/her mouth, if all your other allergy error traps/safety barriers have failed.

The $O$ stands for Ownership. Can you honestly tell me that you treated a place where you lived that you rented to the same standard as one that you owned? From the organisation's executive board members right down to every person working on a ward, medication safety must be treated as if everyone owned it. Two chief pharmacists gave a brilliant illustration of this in the satellite session on automated medicine cabinets. They explained that the finance director was on board as there was a clear plan for the return on investment and that the nurses really bought into the scheme as they were involved in the planning, and it made their (not just pharmacy's) working practices easier and quicker as well as safer. In the other two plenaries, both David Cousins and Howard Bergendahl

Correspondence to Mr Steven D Williams, Department of Pharmacy, University Hospital of South Manchester, Southmoor road, Wythenshawe, Manchester M23 9LT, UK; s.williams@manchester.ac.uk described the value of building a safety culture driven by the workers and managers, as has happened in high-reliability organisations like the nuclear and aviation industries. Pharmacists have much to learn about aiming for the goal of zero preventable deaths due to medicines, but I can tell you one thing for certain, you will not achieve it alone.

That is where the first $M$ comes in, Multidisciplinary. Pharmacists may have the medication expertise and 'attention to detail' attributes to lead the agenda, as David Cousins explained about the UK approach of introducing medication safety officers in all organisations, ${ }^{1}$ but medication safety is not all about pharmacy! We may have the medication expertise and 'attention to detail' attributes to lead the agenda, as explained by David Cousins about the UK approach of introducing medication safety officers in all organisations. ${ }^{1}$ We all know that there is no letter ' $\mathrm{I}$ ' in 'TEAM', and there were plenty of conference sessions and posters about the benefits of teamwork between nurses, doctors and pharmacists to improve medication safety. ${ }^{2}$ We must also remember that transparent collaboration with both the industry and universities to produce strong evidence for the affordability of technologies to improve medication safety, within the current austerity climate, is a multidisciplinary approach too. This was well explained within the lively 'medicine matters, debating the challenges of modern medicine' symposium.

The second $M$ is for Metrics. As sciencebased clinical graduates, I think pharmacists sometimes hide behind Albert Einstein's perceived wise words 'not everything that counts can be counted, and not everything that can be counted counts'. But again, we must take our lead from those high-reliability organisations where it is clear that organisations track real outcome measures, not just process measures. You must then use quality improvement methodologies to track progress if you are going to move towards the goal of zero preventable deaths due to medicines. For example, you would probably know what proportion of patients admitted to your wards had their medicines reconciled by pharmacy within $24 \mathrm{~h}$ of admission, but do you know how many patients had doses of medicines omitted or how many patients were harmed due to a high-risk medicine last month or last year?

Such medication safety metric tools do exist for use in hospitals, but are not yet widely adopted. ${ }^{3}$

The second $P$ is for Positivity. Continually dealing with patient harms or even death due to medication errors can put health professionals into a spiral of negativity. During the conference, I heard of a great positive scheme from a paediatric intensive care unit pharmacist. Health professionals have started reporting when complex events or procedures went really well, and the clinical department then performs a root cause analysis to explain and share 'why' and also to make the multidisciplinary team involved proud of what they achieved! The literature certainly tells us that health professionals are more likely to report safety incidents if there was a positive change in practice afterwards ${ }^{4}$ and that a more positive patient safety culture is associated with fewer adverse events in hospitals. ${ }^{5}$

So, whatever you promised yourself following your Hamburg experience, can I recommend you try using a $P O M M P$ approach to move the medication safety agenda forward in your organisation.

Competing interests None declared.

Provenance and peer review Commissioned; internally peer reviewed.

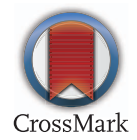

To cite Williams SD. Eur J Hosp Pharm 2015;22:127.

Eur J Hosp Pharm 2015;22:127.

doi:10.1136/ejhpharm-2015-000698

\section{REFERENCES}

1 NHS England. Patient safety alert to improve reporting and learning of medication and medical devices incidents. 2014. http://www.england.nhs.uk/2014/03/ 20/med-devices/

2 Salas E, Sims DE, Klein C, et al. Can team work enhance patient safety? Risk management foundation. Harvard Medical Institutions Forum, 2003. https:// www.rmf.harvard.edu/ /media/Files/_Global/KC/PDFs/ Forum_V23N3_teamworksafety.pdf

3 NHS England. Medication Safety Thermometer. http:// www.safetythermometer.nhs.uk/index.php? option=com_content\&view $=$ article\&id $=3 \&$ Itemid $=107$

4 Leape L. Reporting of adverse events. $N$ Engl J Med 2002;347:1633-8.

5 Mardon RE, Khanna K, Sorra J, et al. Exploring relationships between hospital patient safety culture and adverse events. J Patient Saf 2010;6:226-32. 